\title{
GEOPHYSICAL FLUIDS FROM DIFFERENT DATA SOURCES, GEOMAGNETIC JERKS, AND THEIR IMPACT ON EARTH'S ORIENTATION
}

\author{
Jan VONDRÁK * and Cyril RON \\ Department of Galaxies and Planetary Systems, Astronomical Institute, Czech Academy of Sciences, \\ Bočni II, 14100 Prague 4, Czech Republic \\ *Corresponding author's e-mail: vondrak@ig.cas.cz
}

\begin{tabular}{l} 
ARTICLE INFO \\
\hline Article history: \\
Received 4 December 2015 \\
Accepted 8 February 2016 \\
Available online 2 March 2016 \\
\hline
\end{tabular}

Keywords:

Polar motion

Length-of-day

Free core nutation

Geophysical excitations

Geomagnetic jerks

\begin{abstract}
Recently we studied the effects of geophysical fluids (atmosphere, oceans) and geomagnetic jerks in Earth's orientation in space (Vondrák and Ron, 2010; Ron and Vondrák, 2011). To this end, we used the American NCEP/NCAR model of the atmosphere and ECCO model of the oceans (Vondrák and Ron, 2015). Here we concentrate on other available models of geophysical fluids, such as ERA and MERRA for the atmosphere, and OMCT for the oceans, and compare the results obtained with all of them. We also test the hypothetic effect of geomagnetic jerks together with these alternative models and study how much the agreement with the observed Earth Orientation Parameters is improved. By using numerical integration of all five Earth orientation parameters, we demonstrate that different models of atmospheric/oceanic excitations lead to slightly different results, fitting relatively well with their observed values but showing changes both in amplitude and phase. In all cases the agreement improves substantially when the effect of geomagnetic jerks is added to geophysical fluids, and the differences in amplitude/phase almost disappear.
\end{abstract}

\section{INTRODUCTION}

It was demonstrated earlier (e.g., Lambeck, 1980; Barnes et al., 1983; Brzeziński et al., 2002; Gross et al., 2005) that excitations by geophysical fluids (atmosphere, oceans) play dominant role in polar motion and are responsible (together with tidal effects) also for changes of speed of rotation of the Earth. Later on, it was shown that much smaller, but now observable effect is seen also in nutation (see, e.g., Vondrák and Ron, 2010; Ron and Vondrák, 2011; Ron et al., 2014). However, changes of amplitude and phase of all Earth Orientation Parameters (EOP) exist, when comparing the calculated effects of geophysical fluids with the EOP observations. It was recently shown that these changes occur close to the epochs of geomagnetic jerks (GMJ), rapid changes of the second derivative of intensity of the geomagnetic field (Mandea et al., 2010). Holme and de Viron $(2005,2013)$ demonstrated this for length-of-day changes (LOD), Gibert and Le Mouël (2008) for polar motion, and recently Malkin (2013) for nutation. Very recently we have shown that much better agreement with observations of all five EOP is obtained if additional impulse-like excitations due to GMJ are applied at the epochs of GMJ to the effects of geophysical fluids, calculated from NCEP/NCAR and ECCO models (Vondrák and Ron, 2015). In the following text we further widen the study of these effects, using the excitations by the atmosphere/oceans from different sources, and combine them with GMJ.

\section{THE DATA USED}

In this study, we use the data in the interval twenty years long $(1994.0-2014.0)$, the common interval covered by the time series for all models of excitation, available at the web site of the International Earth Rotation and Reference Systems Service (IERS).

Thus, for the observed polar motion and LOD we use the IERS combined solution C04, which is given in 1-day intervals. For the precession-nutation the differences between the observed values and the models of nutation IAU2000 (Mathews et al., 2002) and precession IAU2006 (Capitaine et al., 2003), in the form of Celestial Pole Offsets - CPO, were used. Namely we use the combined solution of International VLBI Service for Astrometry and Geodesy (IVS) ivs14q1X. eops (Schuh and Behrend, 2012).

The excitations $\chi_{1,2,3}$ due to geophysical fluids (equatorial components $\chi_{1}, \chi_{2}$ influencing polar motion and precession-nutation, axial component $\chi_{3}$ affecting speed of rotation) are available at IERS website:

For the atmosphere, several models from different sources are available: 
- American NCEP/NCAR reanalysis (both with and without Inverted Barometer correction - IB), given in 6-hour steps (Zhou et al., 2006).

- American MERRA (again with and without IB correction), given in 3-hour steps (Schindelegger et al., 2011).

- European ERA interim, in 6-hour steps (Dobslaw et al., 2010).

For the oceans, only two models are available:

- American ECCO model, given in 1-day steps (Gross et al., 2005)

- European OMCT model, given in 6-hour steps (Dobslaw et al., 2010).

It is necessary to say that IB correction represents a simple model of the ocean that reacts immediately and inversely proportionally to the changes of atmospheric pressure (Wunsch and Stammer, 1997).

For geomagnetic jerks, we use only the epochs within the interval studied, i.e., 1999.0, 2003.5, 2004.7, 2007.5 and 2011.0, found in publications by Olsen and Mandea (2008), Mandea et al. (2010), Malkin (2013), and Chulliat and Maus (2014). Similarly to our preceding studies, we apply a bellshaped excitation, $\Delta$ days long, centered at the epochs $t_{0}$ of GMJ, calculated from the expression in complex form

$\chi_{G M J}=\frac{a}{2}\left[1+\cos \frac{2 \pi\left(t-t_{0}\right)}{\Delta}\right]$,

where $\chi=\chi_{1}+i \chi_{2}$ This very form is used because the effect of GMJ is supposed to be impulse-like, acting during a limited time interval (typically several months). On the other hand, it should be a continuous and smooth function, consistent with the character of GMJ. The real shape itself is however not much important - we obtained very similar results in the past, when experimenting with delta or triangle shapes. The excitation of this form leads to only the change of the amplitude and phase of the excited motion, not to the mean value of pole position, as we demonstrated earlier (Vondrák and Ron, 2015), which is in agreement with the observations. This form of GMJ excitation is applied for polar motion and CPO only; in case of LOD its integral, leading to ramp-like form, is used

$$
\Delta L O D_{G M J}=\frac{a}{2} \Delta\left[\frac{t-t_{0}}{\Delta}+0.5+\frac{1}{2 \pi} \sin \frac{2 \pi\left(t-t_{0}\right)}{\Delta}\right] .
$$

We are using here $\Delta=200$ days that is comparable to typical length of GMJ. As follows from our numerous tests made with different values of $\Delta$, 200 days yields the best agreement with the observations. Similarly, we tested the choice of the values of central epochs of the excitation $t_{\mathrm{o}}$ and found that the best results are obtained if they are put equal to the epochs of GMJ. The amplitudes $a$ of these excitations, complex in Eq. (1), real in Eq. (2), are estimated from the condition of the best fit to the observations.

The excitations around the three axes $\chi_{1,2,3}$ due to geophysical fluids are given in terrestrial reference frame, which can be used directly for computing their effect only in polar motion and LOD. However, to calculate their influence in nutation, it is necessary to have them expressed in non-rotating celestial reference frame. In order to do so, we use a simple formula $\chi^{\prime}=-\chi^{i \phi}$, in which $\chi=\chi_{1}+i \chi_{2}$ denotes the complex equatorial component of the excitation in terrestrial frame, $\chi^{\prime}$ the same in celestial frame and $\phi$ the Greenwich sidereal time. Only the longperiodic part of excitation has a measurable effect, the short-periodic excitations being smoothed out during the integration. Thus only the retrograde quasi-diurnal signal in terrestrial frame, which becomes longperiodic in celestial frame, has some influence in nutation. From this follows that sub-diurnal resolution is needed to calculate the effect of geophysical fluids in nutation. This is fulfilled for all atmospheric excitations mentioned above, but from the oceanic models only OMCT can be used; ECCO model (with 1-day resolution) does not fulfill this necessary condition.

\section{CALCULATING GEOPHYSICAL \\ EXCITATIONS}

To calculate geophysical effects in all five EOP, we use the following procedures:

For polar motion in terrestrial frame, $p=x-i y$ ( $x, y$ being the coordinates of instantaneous pole position), Brzeziński's broad-band Liouville equations (Brzeziński, 1994), expressed in complex form, are numerically integrated. They read

$$
\begin{aligned}
& \ddot{p}-i\left(\sigma_{C}+\sigma_{f}\right) \dot{p}-\sigma_{C} \sigma_{f} p= \\
& =-\sigma_{C}\left\{\begin{array}{c}
\sigma_{f}\left(\chi_{p}+\chi_{w}\right)+\sigma_{C}\left(a_{p} \chi_{p}+a_{w} \chi_{w}\right)+ \\
+i\left[\left(1+a_{p}\right) \dot{\chi}_{p}+\left(1+a_{w}\right) \dot{\chi}_{w}\right]
\end{array}\right\},
\end{aligned}
$$

where $\chi_{p}, \chi_{w}$ stand for pressure and wind term, respectively. $\quad \sigma_{C}=0.014602+0.000083 i \quad$ and $\sigma_{f}=-6.31498+0.000153 i$ are the complex resonance Chandler and Free Core Nutation (FCN) frequencies in terrestrial frame, expressed in $\mathrm{rad} / \mathrm{day}$ (Mathews et al., 2002), $a_{p}=9.200 \times 10^{-2}$, $a_{w}=2.628 \times 10^{-4} \quad($ Koot and de Viron, 2011) are dimensionless numerical constants, expressing different reaction of the rotating Earth on pressure and wind excitation, respectively.

For the nutation (celestial pole offsets), $P=d X+i d Y$ in celestial frame, very similar equations hold

$$
\begin{aligned}
& \ddot{P}-i\left(\sigma_{{ }_{C}}+\sigma^{\prime}{ }_{f}\right) \dot{P}-\sigma^{\prime}{ }_{C} \sigma^{\prime}{ }_{f} P= \\
& =-\sigma_{C}\left\{\begin{array}{c}
\sigma_{f}^{\prime}\left(\chi_{p}^{\prime}+\chi^{\prime}{ }_{w}\right)+\sigma^{\prime}{ }_{C}\left(a_{p} \chi^{\prime}{ }_{p}+a_{w} \chi^{\prime}{ }_{w}\right)+ \\
+i\left[\left(1+a_{p}\right) \dot{\chi}_{p}{ }_{p}+\left(1+a_{w}\right) \dot{\chi}^{\prime}{ }_{w}\right]
\end{array}\right\},
\end{aligned}
$$

in which $\sigma_{C}^{\prime}, \sigma_{f}^{\prime}$ are resonance frequencies in celestial frame (there is a simple relation $\sigma^{\prime}=\sigma+\Omega$ between 


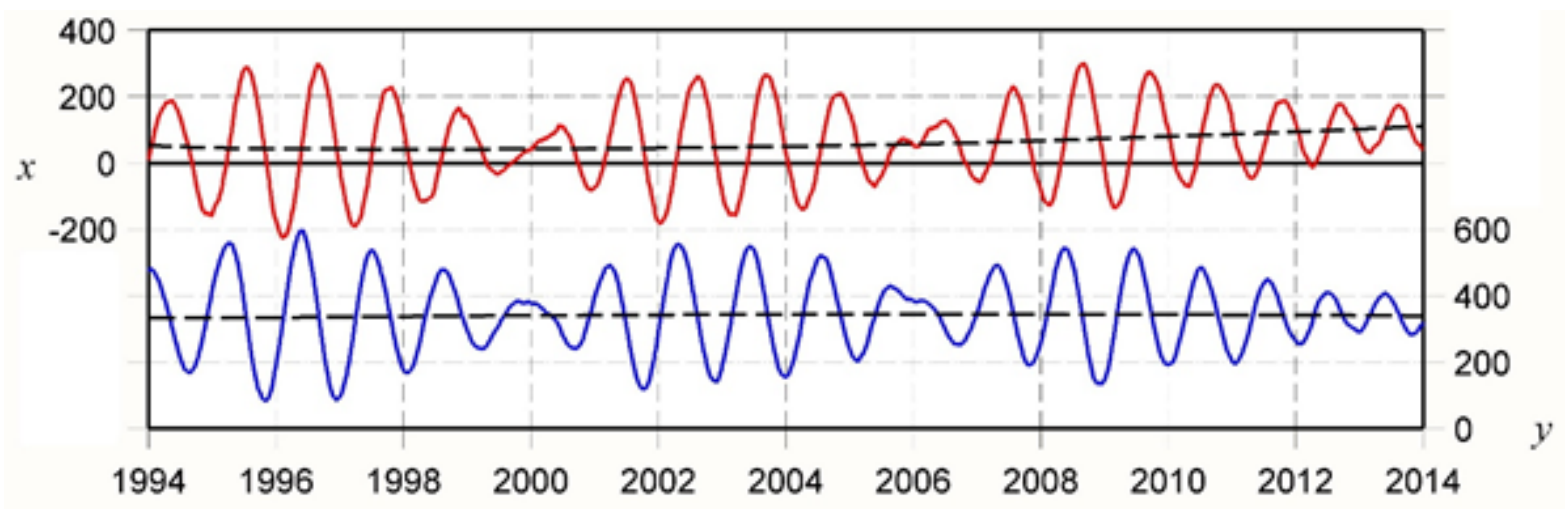

Fig. 1 Components of observed polar motion IERS C04 [mas], and their long-periodic values.

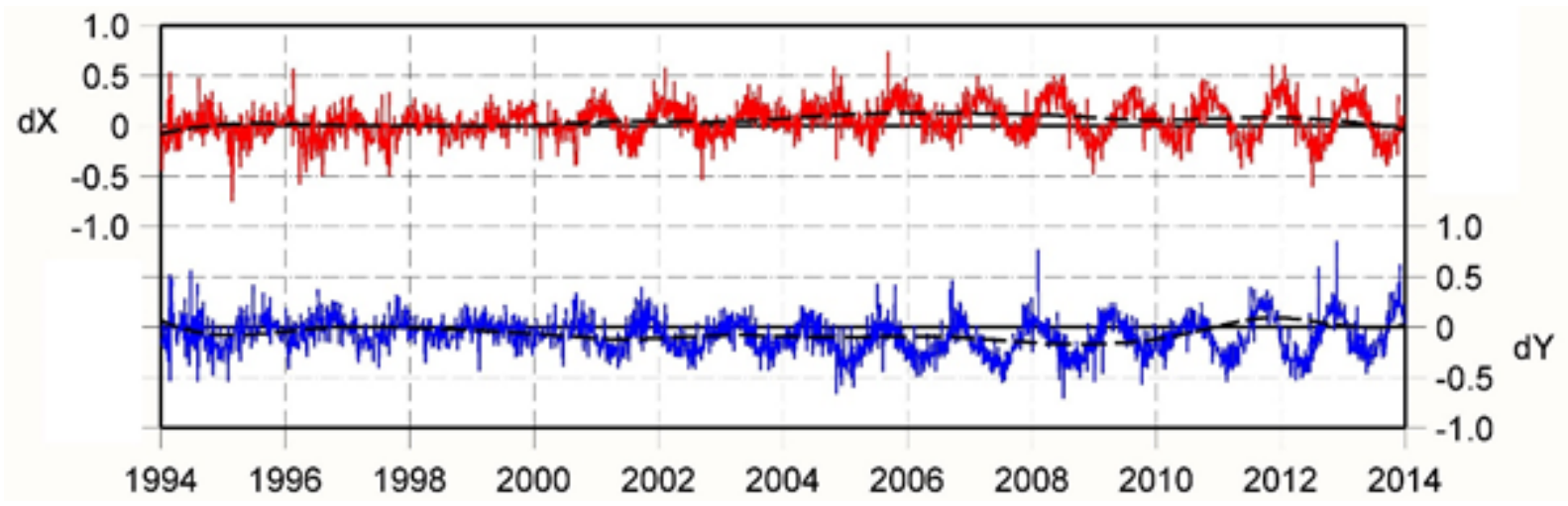

Fig. 2 Components of observed celestial pole offsets IVS [mas], and their long-periodic values.

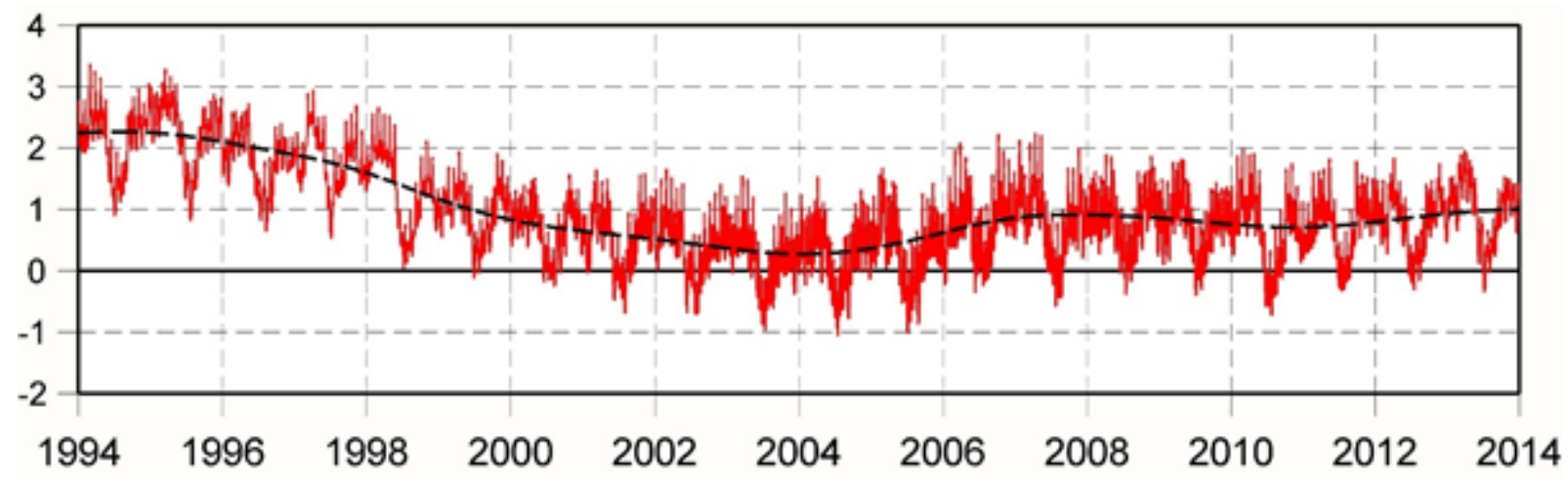

Fig. 3 Observed length-of-day changes IERS C04 [ms], and their long-periodic values.

the values of any frequency in celestial and terrestrial frame, where $\Omega=6.30038 \mathrm{rad} /$ day is the mean speed of Earth's rotation).

To numerically integrate Eqs. (3) and (4), we use traditional fourth-order Runge-Kutta procedure rk4 taken from Press et al. (1992) that we re-wrote into complex form, with 6-hour steps. The initial conditions were chosen so that the integrated pole path fits best to the observations, in a least-squares sense. Both equations describe in general two damped free motions (with frequencies, $\sigma_{C}, \sigma_{f}$ in terrestrial frame, $\sigma_{C}^{\prime}, \sigma_{f}^{\prime}$ in celestial frame), in addition to the forced motion due to excitations. The first free motion is prograde long-periodic and the second one is retrograde quasi-diurnal. Their amplitudes and phases are given by two complex integration constants. Since, by definition, there should be no retrograde quasi-diurnal motion of the pole in either reference frames, we chose only one complex integration constant (initial position of the pole) and constrain the other one so that the free diurnal motion disappears. Prior to comparison, the observed data were smoothed to contain only the periods longer than 10 days, and long-periodic part of the observed polar motion and celestial pole offsets (for periods longer than 16 years) was removed. To this end, we used the filter by Vondrák (1977). The observations and their longperiodic parts are depicted in Figures 1 and 2, in which the vertical scale is given in milliarcseconds (mas). 
Table 1 RMS fit [mas] and correlations between integrated and observed polar motion.

\begin{tabular}{l|llllllllll}
\hline Excitation & \multicolumn{2}{l}{ NCEP IB } & \multicolumn{2}{c}{ NCEP+ECCO } & \multicolumn{2}{c}{ MERRA IB } & \multicolumn{2}{c}{ MERRA+ECCO } & \multicolumn{2}{c}{ ERA+OMCT } \\
& rms & corr. & rms & corr. & rms & corr. & rms & corr. & rms & corr. \\
\hline $\mathbf{A}+\mathbf{O}$ & 59.9 & 0.933 & 78.6 & 0.885 & 56.8 & 0.940 & 72.4 & 0.901 & 41.4 & 0.973 \\
$\mathbf{A}+\mathbf{O}+\mathbf{G}$ & 48.6 & 0.957 & 40.8 & 0.970 & 48.4 & 0.957 & 41.4 & 0.969 & $\mathbf{3 0 . 3}$ & $\mathbf{0 . 9 8 5}$ \\
\hline
\end{tabular}

Table 2 Amplitudes [mas] of additional GMJ excitations in polar motion.

\begin{tabular}{|c|c|c|c|c|c|c|c|c|c|c|}
\hline \multirow[t]{2}{*}{ GMJ epoch } & \multirow{2}{*}{$\begin{array}{c}\text { NCEP IB } \\
\chi_{1} \\
\end{array}$} & & \multicolumn{2}{|c|}{ NCEP+ECCO } & \multicolumn{2}{|c|}{ MERRA IB } & \multicolumn{2}{|c|}{ MERRA+ECCO } & \multicolumn{2}{|c|}{ ERA+OMCT } \\
\hline & & $\chi_{2}$ & $\chi_{1}$ & $\chi_{2}$ & $\chi_{1}$ & $\chi_{2}$ & $\chi_{1}$ & $\chi_{2}$ & $\chi_{1}$ & $\chi_{2}$ \\
\hline 1999.0 & 25 & 0 & 60 & 10 & 30 & -10 & 55 & -5 & 0 & 10 \\
\hline 2003.5 & -40 & 55 & -40 & 50 & -10 & 55 & -5 & 50 & 0 & -15 \\
\hline 2004.7 & 25 & -95 & -30 & -65 & 5 & -80 & -50 & -45 & 60 & -25 \\
\hline 2007.5 & -35 & 0 & -30 & 60 & -35 & 10 & -25 & 75 & 10 & -45 \\
\hline 2011.0 & 15 & -75 & 110 & -60 & 10 & -70 & 105 & -60 & 15 & -15 \\
\hline
\end{tabular}

For LOD the relation between $L O D$ (expressed in seconds of time) and the axial component of the excitations $\chi_{3}$ is simply (Barnes et al., 1983)

$$
L O D=86400\left(\chi_{3 p}+\chi_{3 w}\right)=\text { constant }
$$

The constant part of Eq. (5) is determined to fulfill the condition of the best fit to the observed values. The long-periodic part (for periods longer than 8 years) was removed from the observations, as well as the tidal variations after IERS Conventions (Petit and Luzum, 2010). The observed LOD and their longperiodic part, expressed in milliseconds (ms), are shown in Figure 3.

\section{RESULTS}

All calculations, mentioned in the preceding section, were made in two versions:

a) Excitations only by atmosphere and oceans were used, initial position was determined to lead to the best fit in the whole interval studied, i.e., 1994.0 - 2014.0.

b) Excitations by GMJ are added to the atmospheric and oceanic excitations, initial position was determined to fit to observation in the interval before the epoch of the first GMJ, i.e., 1994.0 1999.0. Then the amplitudes of GMJ excitations were estimated from the best fit condition in each interval between two subsequent GMJ epochs.

\subsection{POLAR MOTION}

Before integrating Eqs. (3), the excitations by geophysical fluids were smoothed to contain only the periods longer than 10 days, and centered (i.e., their mean values subtracted). Their time derivatives $\dot{\chi}$, needed for the integration, were also calculated. The differences between the integrated path of the pole and C04 solution (with long-periodic part removed) for all five atmospheric + oceanic models are depicted in Figure 4, in which GMJ epochs are marked with arrows in the bottom plot. Units of the vertical scale are milliarcseconds (mas). In the upper plot, the solutions for only geophysical fluids are shown, in the lower one GMJ excitations are added. It is clear that the deviations diminish, and the largest ones completely disappear, if GMJ excitations are applied, for all models of the atmosphere/oceans. Nevertheless, from the figure we can also see that there still remain small residual deviations with quasi-annual period. A detailed information on the root-mean-square fits and correlations of all individual solutions is summarized in Table1. The best fit from all these integrations is evidently obtained for ERA + OMCT + GMJ model of excitations.

Amplitudes of additional excitations due to GMJ, whose values were estimated to yield the best fit to observed polar motion, are given in Table 2 .

\subsection{CELESTIAL POLE OFFSETS}

These are obtained from integrating Eqs. (4); excitations had to be first recomputed into celestial reference frame, centered, smoothed and their time derivatives computed. The results are graphically displayed in Figure 5, where differences from IVS combined solution are given. Before computing these differences, IVS CPO values were filtered to contain only the periods between 60 and 6000 days, and the Sun-synchronous correction with annual period, applied by Mathews et al. (2002) to account for the missing atmospheric effect in IAU2000 model of nutation. Since ECCO oceanic model cannot be used (see Section 2), only three solutions were made. Vertical scale is given in mas; notice that the differences are about two orders of magnitude smaller than for polar motion (compare Figs. 4 and 5). Again, the upper plot shows the integration with only atmospheric and oceanic excitations, the lower one with additional impulse-like excitations at GMJ epochs (arrows in lower plot) applied. It is clear that the deviations with approximately FCN period (of about 430 days) in upper plot practically disappear in lower plot, where GMJ excitations are added. 
Table 3 RMS fit [mas] and correlations between integrated and observed celestial pole offsets.

\begin{tabular}{l|llllll}
\hline Excitation & \multicolumn{2}{|c}{ NCEP IB } & \multicolumn{2}{c}{ MERRA IB } & \multicolumn{2}{c}{ ERA+OMCT } \\
& rms & corr. & rms & corr. & rms & corr. \\
\hline $\mathbf{A}+\mathbf{O}$ & 0.209 & 0.411 & 0.225 & 0.270 & 0.311 & 0.069 \\
\cline { 2 - 7 } $\mathbf{A}+\mathbf{O}+\mathbf{G}$ & $\mathbf{0 . 1 4 6}$ & $\mathbf{0 . 7 6 6}$ & 0.167 & 0.681 & 0.207 & 0.619 \\
\hline
\end{tabular}

Root-mean-square values of the fits and correlations are displayed in Table 3. Unlike in polar motion, the best fit and largest correlation are obtained in case of integration with NCEP IB excitations.

Amplitudes of additional excitations due to GMJ, whose values were estimated to yield the best fit to observed celestial pole offsets, are given in Table 4 .

\subsection{LENGTH-OF-DAY}

It is well known that LOD changes are caused not only by atmospheric and oceanic excitations, but also by tidal deformations of the Earth. There are also decadal variations of not very well identified origin (probably due to core-mantle interaction). Therefore the part caused by tidal effects, as well as longperiodic part were removed from the observed values (as described above in Section 3), before comparing them with the changes computed from Eq. (5). The results are shown in Figure 6, in which the vertical scale is expressed in milliseconds of time. Similarly to polar motion and celestial pole offsets, the fit is improved when excitations at GMJ epochs are applied. However, there are rather large peaks in the residuals around 1997.5 (positive) and 2000.5 (negative). They are more or less the same for all models of excitations, but these are not reflected in variations of LOD. Applying GMJ excitations diminishes their amplitudes only partially. It seems that there exist some additional source of excitation, requiring further study.

Overview of root-mean-square deviations and correlations between integrated and observed LOD is
Table 4 Amplitudes [mas] of additional GMJ excitations in celestial pole offsets.

\begin{tabular}{c|rrrrrr}
\hline & \multicolumn{2}{|c}{ NCEP IB } & \multicolumn{3}{c}{ MERRA IB } & \multicolumn{2}{c}{ ERA+OMCT } \\
GMJ & $\chi_{1}$ & $\chi_{2}$ & $\chi_{1}$ & $\chi_{2}$ & $\chi_{1}$ & $\chi_{2}$ \\
epoch & & & & & & \\
\hline 1999.0 & -0.45 & 0.80 & -0.70 & 1.05 & -3.35 & -0.55 \\
2003.5 & 0.70 & -1.15 & 0.85 & -0.90 & 1.15 & -1.45 \\
2004.7 & 0.25 & -0.75 & 0.05 & -0.95 & 0.20 & -0.05 \\
2007.5 & -1.10 & -1.45 & -0.65 & -1.00 & -0.70 & -0.20 \\
2011.0 & -0.65 & -0.35 & -1.00 & -0.60 & -1.20 & -0.90 \\
\hline
\end{tabular}

given in Table 5. In this case, the best fit and correlation is obtained for MERRA + ECCO solution. The differences between IB and ECCO oceanic modeling are relatively small, probably because the influence of the ocean on speed of rotation is very small, if compared with the atmosphere (where the effect of zonal winds is dominant).

The amplitudes of additional GMJ excitations, leading to the best fit between computed and observed LOD, are displayed in Table 6.

\section{CONCLUSIONS}

Geophysical excitations play a significant role in all EOP, however we demonstrate that the results, based on excitations by geophysical fluids only (atmosphere, oceans), do not provide fully satisfactory agreement with the observations. Different atmospheric/oceanic models yield only slightly different results. Most of the discrepancies in all EOP almost disappear if additional impulse-like excitations are applied at GMJ epochs. This holds for all models of atmospheric and oceanic excitations used in this study. The best fit and correlation is however achieved for different parameters with different solutions:

- For polar motion, it is ERA + OMCT + GMJ;

- For LOD, it is MERRA + ECCO + GMJ;

- For celestial pole offsets, it is NCEP IB + GMJ.

Table 5 RMS fit [ms] and correlations between integrated and observed LOD.

\begin{tabular}{l|llllllllll}
\hline Excitation & \multicolumn{2}{|l}{ NCEP IB } & \multicolumn{2}{c}{ NCEP+ECCO } & \multicolumn{2}{c}{ MERRA IB } & \multicolumn{2}{c}{ MERRA+ECCO } & \multicolumn{2}{c}{ ERA+OMCT } \\
& rms & corr. & rms & corr. & rms & corr. & rms & corr. & rms & corr. \\
\hline $\mathbf{A}+\mathbf{O}$ & .110 & 0.957 & .110 & 0.957 & .093 & 0.969 & .085 & 0.974 & .112 & 0.955 \\
$\mathbf{A}+\mathbf{O}+\mathbf{G}$ & .096 & 0.967 & .095 & 0.967 & .084 & 0.975 & $\mathbf{. 0 7 8}$ & $\mathbf{0 . 9 7 9}$ & .103 & 0.962 \\
\hline
\end{tabular}

Table 6 Amplitudes [ $\mu \mathrm{s} /$ day] of additional GMJ excitations in LOD.

\begin{tabular}{c|rcrrr}
\hline GMJ epoch & NCEP IB & NCEP+ECCO & MERRA IB & MERRA+ECCO & ERA+OMCT \\
\hline 1999.0 & 0.84 & 0.87 & 0.65 & 0.60 & 0.65 \\
2003.5 & -0.57 & -0.58 & -0.48 & -0.49 & -0.48 \\
2004.7 & 0.37 & 0.40 & 0.26 & 0.30 & 0.26 \\
2007.5 & -0.01 & -0.04 & 0.03 & -0.01 & 0.03 \\
2011.0 & 0.00 & -0.02 & 0.02 & 0.00 & 0.02 \\
\hline
\end{tabular}


Even for these best fits there still remain small systematic residuals that cannot be explained by a combined effect of the atmosphere, oceans and geomagnetic jerks. In case of polar motion, periodic variations with quasi-annual period, in case of LOD two dominant peaks around 1997.5 and 2002.5. These variations require further study. It also should be noted that the improvement of the fit with GMJ excitations is somewhat less significant in LOD case.

\section{ACKNOWLEDGEMENTS}

This research was financially supported by the grant No. 13-15943S "Geophysical excitations in the motion of Earth's axis of rotation", awarded by the Grant Agency of the Czech Republic. The authors express their thanks to Michael Schindelegger (TU Vienna), who kindly provided the atmospheric angular momentum functions based on MERRA data.

\section{REFERENCES}

Barnes, R.T.H., Hide, R., White, A.A. and Wilson, C.A.: 1983, Atmospheric angular momentum fluctuations, length-of-day changes and polar motion. Proc. R. Soc. Lond., A 387, 31-73.

Brzeziński, A.: 1994, Polar motion excitation by variations of the effective angular momentum function: II. Extended Model. Manuscripta Geodaetica, 19, 157171.

Brzeziński, A., Bizouard, C. and Petrov, S.: 2002, Influence of the atmosphere on Earth rotation: what new can be learned from the recent atmospheric angular momentum estimates? Surveys in Geophysics, 23, 3369. DOI: $10.1023 / \mathrm{A}: 1014847319391$

Capitaine, N., Wallace, P.T. and Chapront, J.: 2003, Expressions for IAU 2000 precession quantities. Astron. Astrophys., 412, 567-586. DOI: 10.1051/0004-6361:20031539

Chulliat, A. and Maus, S.: 2014, Geomagnetic secular acceleration, jerks, and a localized standing wave at the core surface from 2000 to 2010. J. Geophys. Res. Solid Earth, 119, 1531. DOI: 10.1003/2013B010604

Dobslaw, H., Dill, R., Grotzsch, A., Brzezinski, A. and Thomas, M.: 2010, Seasonal polar motion excitation from numerical models of atmosphere, ocean, and continental hydrosphere. J. Geophys. Res., 115, B10406. DOI: 10.1029/2009B007127

Gibert, D. and Le Mouël, J.-L.: 2008, Inversion of polar motion data: Chandler wobble, phase jumps, and geomagnetic jerks. J. Geophys. J., 113, B10405. DOI: $10.1029 / 2008 J B 005700$

Gross, R.S., Fukumori, I. and Menemenlis, D.: 2005, Atmospheric and oceanic excitation of decadal-scale Earth orientation variations. J. Geophys. Res., 110, B09405. DOI: 10.1029/2004JB003565

Holme, R. and de Viron, O.: 2005, Geomagnetic jerks and a high-resolution length-of-day profile for core studies. Geophys. J. Int., 160, 435-439. DOI: $10.1111 / \mathrm{j} .1365-246 X .2004 .02510 . \mathrm{x}$

Holme, R. and de Viron, O.: 2013, Characterization and implications of intradecadal variations in length of day. Nature, 499, No. 7457, 202-205.

DOI: 10.1038 /nature 12282
Koot, L. and de Viron, O.: 2011, Atmospheric contribution to nutation and implications for the estimation of deep Earth's properties from nutation observations. Geophys. J. Int., 185, 1255-1265. DOI: $10.1111 / \mathrm{j} .1365-246$ X.2011.05026.x

Lambeck, K.: 1980, The Earth's variable rotation: Geophysical causes and consequences. Cambridge Univ. Press.

Malkin, Z.: 2013, Free core nutation and geomagnetic jerks. J. Geodyn., 72, 53-58. DOI: $10.1016 /$ j.jog.2013.06.001

Mandea, M., Holme, R., Pais, A., Pinheiro, K., Jackson, A. and Verbanac, G.: 2010, Geomagnetic jerks: Rapid core field variations and core dynamics. Space Sci. Rev., 155, 147-175. DOI: $10.1007 / \mathrm{s} 11214-010-9663-\mathrm{x}$

Mathews, P.M., Herring, T.A. and Buffet, B.A.: 2002, Modeling of nutation-precession for nonrigid Earth, and insights into the Earth's interior. J. Geophys. Res., 107, B4. DOI: 10.1029/2001JB000390

Olsen, N. and Mandea, M.: 2008, Rapidly changing flows in the Earth's core. Nature Geosci., 1, 390-394. DOI: $10.1038 /$ ngeo203

Petit, G. and Luzum, B.: 2010, IERS Conventions 2010. IERS Tech. Note, 36, Ver. Bundesamts für Kartografie und Geodäsie, Frankfurt a.M.

Press, W.H., Teukolsky, S.A., Vetterling and W.T, Flannery, B.P.: 1992, Numerical Recipes in Fortran 77, The Art of Scientific Computing, 2nd Edition. Cambridge University Press.

Ron, C. and Vondrák, J.: 2011, Coherence between geophysical excitations and celestial pole offsets. Acta Geodyn. Geomat., 8, No. 3, 243-247.

Ron, C., Vondrák, J. and Chapanov, Ya.: 2014, Free core nutation - possible causes of changes of its phase and amplitude. In: N. Capitaine (ed.) Proc. Journées 2013 Systèmes de référence spatio-temporels, Observatoire de Paris, 164-167.

Schindelegger, M., Böhm, J., Salstein, D. and Schuh H.: 2011, High-resolution atmospheric angular momentum functions related to Earth rotation parameters during CONT08. J. Geod., 85, 425-433. DOI: $10.1007 / \mathrm{s} 00190-011-0458-\mathrm{y}$

Schuh, H. and Behrend, D.: 2012, VLBI: A fascinating technique for geodesy and astrometry. J. Geodyn., 61, 68-80. DOI: 10.1016/j.jog.2012.07.007

Vondrák, J.: 1977, Problem of smoothing of observational data II. Bull. Astron. Inst. Czechosl., 28, 84-89.

Vondrák, J. and Ron, C.: 2010, Study of atmospheric and oceanic excitations in the motion of Earth's spin axis in space. Acta Geodyn. Geomater., 7, No. 1, 19-28.

Vondrák, J. and Ron, C.: 2015, Earth orientation and its excitations by atmosphere, oceans, and geomagnetic jerks. Serb. Astron. J., 191, 59-66. DOI: $10.2298 / \mathrm{SAJ} 1591059 \mathrm{~V}$

Wunsch, C. and Stammer, D.: 1997, Atmospheric loading and the oceanic "inverted barometer" effect. Reviews of Geophysics, 35, 79-107.

Zhou, Y.H., Salstein, D.A. and Chen, J.L.: 2006, Revised atmospheric excitation function series related to Earth variable rotation under consideration of surface topography. J. Geophys. Res., 111, D12108. DOI: 10.1029/2005JD006608000000000 

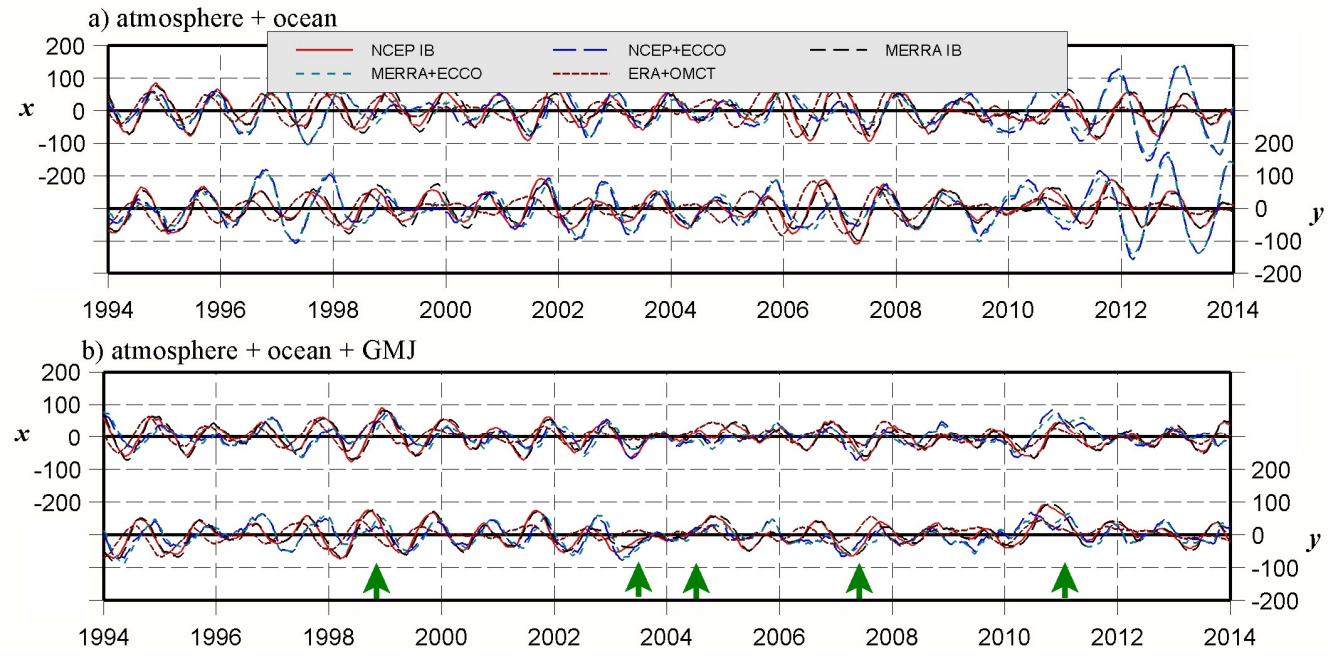

Fig. 4 Differences between the integrated and observed polar motion [mas].
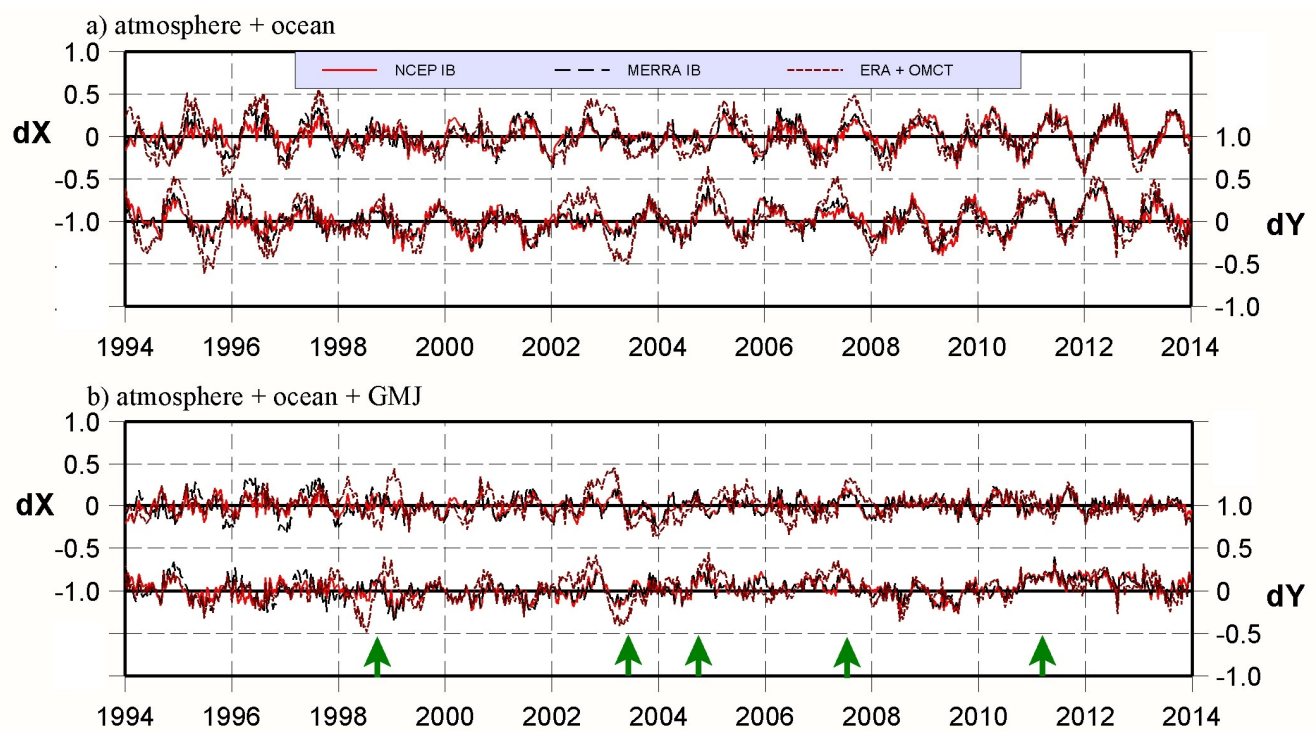

Fig. 5 Differences between integrated and observed celestial pole offsets [mas].

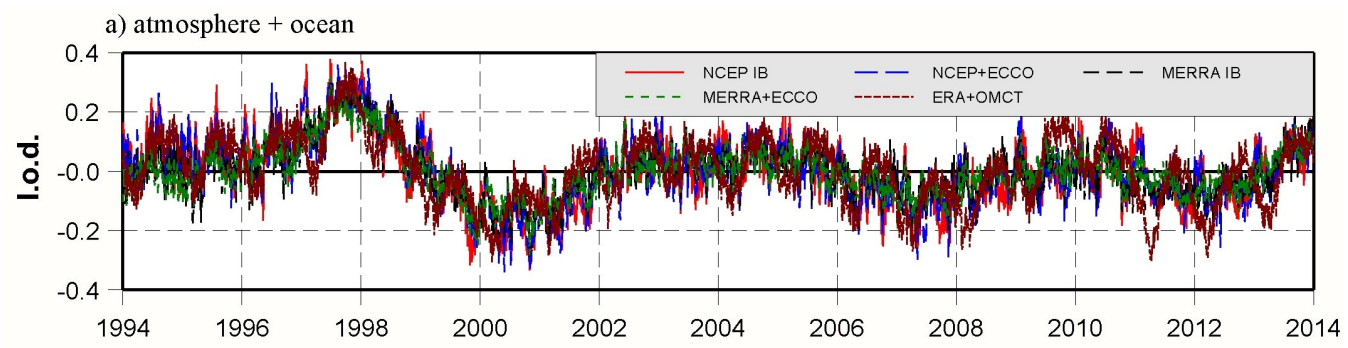

b) atmosphere + ocean + GMJ

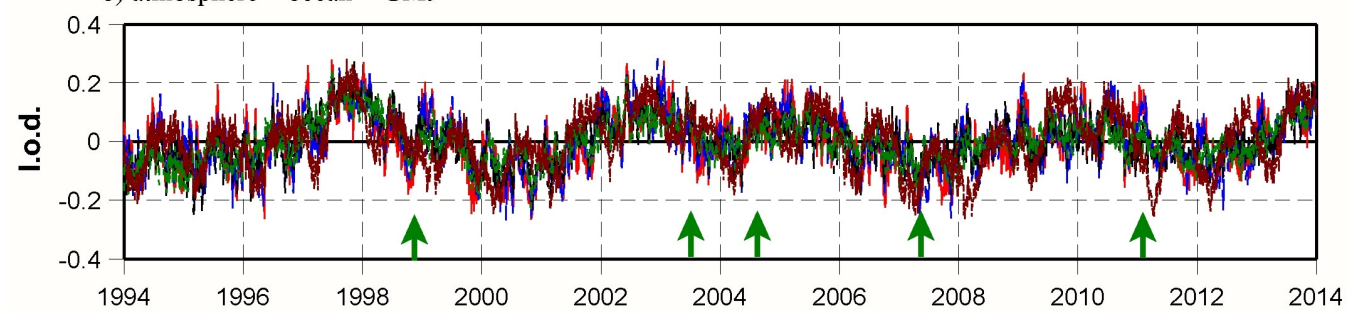

Fig. 6 Differences between calculated and observed variations of length-of-day [ms]. 